\title{
A Remark on the Singular Cauchy Problem, for all Values of the Time, for the Euler-Poisson-Darboux Equation
}

\section{J. B. DIAZ* \& ABDUL R. KIWAN}

Abstract. The purpose of the present note is to simplify the treatment of J. B. Diaz and G. S. S. Ludford for obtaining a solution, for all values of the time, to the singular Cauchy problem for the Euler-Poisson-Darboux equation for values of the parameter $k>m-1$. It is verified directly that a certain multiple integral involving the initial value function $f\left(x_{1}, x_{2}, \cdots x_{m}\right)$ provides a solution to the problem. The treatment given here is a modification of an argument due to A. Weinstein. Weinstein's proof requires that $f$ be of class $C^{3}$, while the present discussion requires only that $f$ be of class $C^{2}$.

1. Introduction. The singular second order partial differential equation

$$
\Delta u=\frac{\partial^{2} u}{\partial t^{2}}+\frac{k}{t} \frac{\partial u}{\partial t}
$$

where $k$ is a parameter,

$$
\Delta=\frac{\partial^{2}}{\partial x_{1}^{2}}+\frac{\partial^{2}}{\partial x_{2}^{2}}+\cdots+\frac{\partial^{2}}{\partial x_{m}^{2}}
$$

is the Laplacian, and $u\left(x_{1}, x_{2}, \cdots, x_{m}, t\right)$ is a real valued function, is known as the Euler-Poisson-Darboux equation (abbreviated EPD). This singular partial differential equation has occurred, for special values of $k$ and $m$, in many important and classical problems since the time of Euler.

The singular Cauchy problem (one sided, i.e., for $t>0$ ) for the EPD equation requires the determination of a real valued function $u\left(x_{1}, \cdots, x_{m}, t\right)$ which satisfies (1) for $t>0$, and meets the initial conditions

$$
u\left(x_{1}, \cdots, x_{m}, 0\right)=f\left(x_{1}, \cdots, x_{m}\right), \quad \frac{\partial u}{\partial t}\left(x_{1}, \cdots, x_{m}, 0\right)=0,
$$

* The research of this author was supported by Grant AFOSR 400-64 from the Air Force Office of Scientific Research to the University of Maryland and by Grant AFOSR 1122-66 to the University of California, Riverside, which is his present address. 
where $f$ is a given real valued function defined for all values of the real variables $x_{1}, \cdots, x_{m}$. (When $k=0$ the EPD equation (1) reduces to the wave equation in $m$ space dimensions; while for $k \neq 0$ the coefficient $k t^{-1}$ is infinite on the plane $t=0$, the carrier of the Cauchy data.) For $k$ any real number, this singular Cauchy problem (1), (2) was first solved by A. Weinstein [1], [2], who employed what he termed the "method of recurrence" and a generalized method of descent. Weinstein's method is based upon the known solution of the (one sided) Cauchy problem (1), (2) for $k=m-1$ :

$$
u(x, t)=\frac{1}{\omega_{m}} \iint_{\sum_{i=1}^{m} \alpha_{i^{2}-1}} f(x+t \alpha) d \omega_{m},
$$

where $x=\left(x_{1}, \cdots, x_{m}\right), \alpha=\left(\alpha_{1}, \cdots, \alpha_{m}\right), d \omega_{m}$ is the surface element of the unit sphere in $m$ dimensional Euclidean space, and

$$
\omega_{m}=\frac{2 \pi^{m / 2}}{\Gamma\left(\frac{m}{2}\right)}
$$

is the surface area of this sphere; and on the explicit formula

$$
u(x, t)=\frac{\omega_{k+1-m}}{\omega_{k+1}} \iint_{\sum_{i=1}^{m} \alpha_{i}<<1} f(x+\alpha t)\left(1-\alpha^{2}\right)^{(k-m-1) / 2} d \alpha,
$$

where $\alpha^{2}=\sum_{i=1}^{m} \alpha_{i}^{2}$ and $d \alpha=d \alpha_{1} \cdots d \alpha_{m}$, for the solution of the problem (1), (2) for $k>m-1$.

J. B. Diaz and H. F. Weinberger [3] obtained a solution of the same Cauchy problem (1), (2), using again as a starting point the same explicit formula (4) for the solution for $k>m-1$ which was employed by Weinstein, and then continuing this definite integral (4) analytically with respect to the parameter $k$.

The singular Cauchy problem (two sided, i.e., for $-\infty<t<+\infty$ ) for the EPD equation (1) consists in the determination of a real valued function $u\left(x_{1}, \cdots, x_{m}, t\right)$ which satisfies (1) for $t \neq 0$, satisfies the initial conditions (2), and which, on the singular plane $t=0$, satisfies "the limiting form, as $t$ approaches zero, of (1)," namely

$$
\Delta u=(k+1) \frac{\partial^{2} u}{\partial t^{2}} .
$$

This problem was formulated by J. B. Diaz and G. S. S. Ludford [4]. The method of solution of (1), (2), for $-\infty<t<+\infty$, given in [4], is based upon showing that the formula (4) yields a solution, for all time, for $k>m-1$. This is done (see p. 78 of [4]), by first transforming the definite integral with fixed limits of integration appearing in (4) to an integral with variable limits of integration (see equation (17) on p. 78 of [4]). 
The purpose of the present note is to simplify the treatment of [4] by verifying directly that the formula (4) gives a solution of the singular Cauchy problem (1), (2), for all time, for $k>m-1$, thus avoiding all the possible complications arising from the detailed justification of the differentiation of a multiple integral with variable limits of integration, such as that in equation (17) on page 78 of [4]. The direct verification for $k>m-1$ given here is a modification of that given by Weinstein in section 6 of [2]. However, Weinstein's argument requires that $f$ be of class $C^{3}$, while the present argument requires only that $f$ be of class $C^{2}$. Intuitively speaking, the present verification bears the same relation to Weinstein's verification as Du Bois Reymond's Lemma bears to the "Fundamental Lemma" in the calculus of variations (see e.g., Bolza [5, pp. 20-25]).

2. Verification for $k>m-1$. Formula (4) may be written:

$$
u(x, t)=\frac{\Gamma\left(\frac{k+1}{2}\right)}{\pi^{m / 2} \Gamma\left(\frac{k+1-m}{2}\right)} \iint_{\sum_{i=1}^{m} \alpha_{i}^{*}<1} f(x+\alpha t)\left(1-\alpha^{2}\right)^{(k-m-1) / 2} d \alpha,
$$

where the integral in (6) converges for all $k>m-1$. Furthermore, if, given $f$, one defines $u(x, t)$ by means of the right hand side of $(6)$ for these values of $k$ and all values of $(x, t)$, then $u(x, t) \varepsilon C^{2}(x, t)$ whenever $f \varepsilon C^{2}(x)$. In fact, if $f \varepsilon C^{r}(x)$, then $u(x, t) \varepsilon C^{r}(x, t)$ and is an even function of $t$. Putting $t=0$ in (6), and performing the integration, one obtains:

i.e.,

$$
u(x, 0)=\frac{\Gamma\left(\frac{k+1}{2}\right)}{\pi^{m / 2} \Gamma\left(\frac{k+1-m}{2}\right)} \int_{0}^{1} f(x) \omega_{m}\left(1-\rho^{2}\right)^{(k-m-1) / 2} \rho^{m-1} d \rho,
$$

$$
u(x, 0)=f(x) .
$$

Furthermore, since $u(x, t) \varepsilon C^{2}(x, t)$, and $u$ is an even function of $t$,

$$
u_{t}(x, 0)=0 \text {. }
$$

Thus, the initial conditions (2) are satisfied. It remains to verify that, for $t \neq 0$, (1) is satisfied, and that for $t=0$ the limiting form (5) holds. Let

$$
v(x, t)=\iint_{\sum_{i=1}^{m} \alpha_{i}<<1} f(x+\alpha t)\left(1-\alpha^{2}\right)^{(k-m-1) / 2} d \alpha,
$$

hence

$$
u(x, t)=\frac{\Gamma\left(\frac{k+1}{2}\right)}{\pi^{m / 2} \Gamma\left(\frac{k+1-m}{2}\right)} v(x, t) .
$$


Differentiating (7) with respect to $t$ gives

$$
v_{t}(x, t)=\sum_{i=1}^{m} \iint_{\alpha^{2}<1} f_{i} \cdot \alpha_{i}\left(1-\alpha^{2}\right)^{(k-m-1) / 2} d \alpha,
$$

where

$$
f_{i}=\frac{\partial f}{\partial x_{i}}=\frac{1}{t} \frac{\partial f}{\partial \alpha_{i}} \quad t \neq 0 .
$$

Letting $R^{2}=\alpha^{2}-\alpha_{i}^{2}$, and integrating (8) by parts with respect to $\alpha_{i}$, one obtains

$$
\begin{aligned}
& v_{t}(x, t)=\frac{1}{t} \sum_{i=1}^{m} \iint_{R^{2}<1} d \alpha_{1} \cdots d \alpha_{i-1} d \alpha_{i+1} \cdots d \alpha_{m} \int_{-\sqrt{1-R^{2}}}^{\sqrt{1-R^{2}}} \frac{\partial f}{\partial \alpha_{i}} \alpha_{i}\left(1-\alpha^{2}\right)^{(k-m-1) / 2} d \alpha_{i} \\
& =\frac{1}{t} \sum_{i=1}^{m} \iint_{R^{2}<1} d \alpha_{1} \cdots d \alpha_{i-1} d \alpha_{i+1} \cdots d \alpha_{m}\left[\left.f \alpha_{i}\left(1-\alpha^{2}\right)^{(k-m-1) / 2}\right|_{\alpha_{i}=-\sqrt{1-R^{2}}} ^{\alpha_{i}=\sqrt{1-R^{2}}}\right. \\
& \left.-\int_{-\sqrt{1-R^{2}}}^{\sqrt{1-R^{2}}} f \cdot\left(1-\alpha^{2}\right)^{(k-m-1) / 2} d \alpha_{i}+(k-m-1) \int_{-\sqrt{1-R^{2}}}^{\sqrt{1-R^{2}}} f \cdot \alpha_{i}^{2}\left(1-\alpha^{2}\right)^{(k-m-3) / 2} d \alpha_{i}\right] .
\end{aligned}
$$

For $k>m+1$, the "boundary" integrals vanish, and one gets

$v_{t}(x, t)=-\frac{m}{t} \iint_{\alpha^{2}<1} f\left(1-\alpha^{2}\right)^{(k-m-1) / 2} d \alpha+\frac{k-m-1}{t} \iint_{\alpha^{2}<1} f \alpha^{2}\left(1-\alpha^{2}\right)^{(k-m-3) / 2} d \alpha$.

Substituting $-\left[\left(1-\alpha^{2}\right)-1\right]$ for $\alpha^{2}$ in the second integral, and using (7), one obtains

$$
\text { (9) } \quad v_{t}(x, t)=-\frac{k-1}{t} v(x, t)+\frac{k-m-1}{t} \iint_{\alpha^{2}<1} f\left(1-\alpha^{2}\right)^{(k-m-3) / 2} d \alpha \text {. }
$$

Another differentiation with respect to $t$ gives

$$
\begin{array}{r}
v_{t t}(x, t)=\frac{k-1}{t^{2}} v(x, t)-\frac{k-1}{t} v_{t}(x, t)-\frac{k-m-1}{t^{2}} \iint_{\alpha^{2}<1} f \cdot\left(1-\alpha^{2}\right)^{(k-m-3) / 2} d \alpha \\
+\frac{k-m-1}{t} \iint_{\alpha^{*}<1} \sum_{i=1}^{m} f_{i} \alpha_{i}\left(1-\alpha^{2}\right)^{(k-m-3) / 2} d \alpha .
\end{array}
$$

Using (9), it follows that

$$
v_{t t}(x, t)+\frac{k}{t} v_{t}(x, t)=\sum_{i=1}^{m} \frac{k-m-1}{t} \iint_{\alpha^{2}<1} f_{i} \alpha_{i}\left(1-\alpha^{2}\right)^{(k-m-3) / 2} d \alpha .
$$

This equation may be rewritten thus 
$v_{t t}+\frac{k}{t} v_{t}=-\sum_{i=1}^{m} \frac{1}{t} \iint_{\alpha^{2}<1} \frac{\partial f}{\partial x_{i}} \cdot \frac{\partial}{\partial \alpha_{i}}\left(1-\alpha^{2}\right)^{(k-m-1) / 2} d \alpha$

$=-\sum_{i=1}^{m} \frac{1}{t} \iint_{R^{2}<1} d \alpha_{1} \cdots d \alpha_{i-1} d \alpha_{i+1} \cdots d \alpha_{m} \int_{-\sqrt{1-R^{2}}}^{\sqrt{1-R^{2}}} \frac{\partial f}{\partial x_{i}} \frac{\partial}{\partial \alpha_{i}}\left(1-\alpha^{2}\right)^{(k-m-1) / 2} d \alpha_{i}$.

An integration by parts with respect to $\alpha_{i}$ yields

$$
\begin{aligned}
v_{t t}+\frac{k}{t} v_{t}=-\sum_{i=1}^{m} \frac{1}{t} \iint_{R^{2}<1} d \alpha_{1} \cdots d \alpha_{i-1} d \alpha_{i+1} \cdots d \alpha_{m} \\
\cdot\left[\left.\frac{\partial f}{\partial x_{i}}\left(1-\alpha^{2}\right)^{(k-m-1) / 2}\right|_{-\sqrt{1-R^{2}}} ^{\sqrt{1-R^{2}}}-\int_{-\sqrt{1-R^{2}}}^{\sqrt{1-R^{2}}} \frac{\partial^{2} f}{\partial \alpha_{i} \partial x_{i}} \cdot\left(1-\alpha^{2}\right)^{(k-m-1) / 2} d \alpha_{i}\right] .
\end{aligned}
$$

Again, the "boundary" integrals vanish, and one obtains

$$
v_{t t}+\frac{k}{t} v_{t}=\sum_{i=1}^{m} \iint_{\alpha^{2}<1} \frac{1}{t} \frac{\partial^{2} f}{\partial \alpha_{i} \partial x_{i}}\left(1-\alpha^{2}\right)^{(k-m-1) / 2} d \alpha .
$$

However, since

$$
\frac{1}{t} \frac{\partial^{2} f}{\partial \alpha_{i} \partial x_{i}}=\frac{\partial^{2} f}{\partial x_{i}^{2}}
$$

one obtains

$$
v_{t t}+\frac{k}{t} v_{t}=\sum_{i=1}^{m} \iint_{\alpha^{2}<1} \frac{\partial^{2} f}{\partial x_{i}^{2}}\left(1-\alpha^{2}\right)^{(k-m-1) / 2} d \alpha
$$

i.e.,

$$
v_{t t}+\frac{k}{t} v_{t}=\Delta v
$$

But,

$$
u(x, t)=\frac{\Gamma\left(\frac{k+1}{2}\right)}{\pi^{m / 2} \Gamma\left(\frac{k+1-m}{2}\right)} v(x, t),
$$

hence

$$
-\Delta u(x, t)+\frac{\partial^{2} u(x, t)}{\partial t^{2}}+\frac{k}{t} \frac{\partial u(x, t)}{\partial t}=0 .
$$

Thus, it follows from (6) that the function $\left(-\Delta+\partial^{2} / \partial t^{2}+(k / t)(\partial / \partial t) u(x, t)\right.$ is continuous for all $(x, t)$ with $t \neq 0$ and that for any fixed $(x, t)$ with $t \neq 0$, the function $\left(-\Delta+\partial^{2} / \partial t^{2}+(k / t)(\partial / \partial t)\right) u(x, t)$ is analytic in $k$ for $k>m-1$. 
Since, however, this function vanishes for $k>m+1$, it follows that

$$
\left(-\Delta+\frac{\partial^{2}}{\partial t^{2}}+\frac{k}{t} \frac{\partial}{\partial t}\right) u(x, t)=0
$$

for all $k>m-1$, in view of the analyticity in $k$. Consider now $t=0$. One has that

$$
\lim _{t \rightarrow 0}\left(-\Delta+\frac{\partial^{2}}{\partial t^{2}}+\frac{k}{t} \frac{\partial}{\partial t}\right) u(x, t) \quad(t \neq 0)
$$

exists, because $u(x, t) \varepsilon C^{2}(x, t)$ for all $(x, t)$, and also that (see (2)):

$$
\lim _{t \rightarrow 0} \frac{k}{t} \frac{\partial u(x, t)}{\partial t}=k \frac{\partial^{2} u(x, 0)}{\partial t^{2}} \quad(t \neq 0) .
$$

Hence, the limit of (11) as $t \rightarrow 0$ is:

$$
-\Delta u(x, 0)+(k+1) u_{t t}(x, 0)=0 .
$$

Thus, it has been proved that if $k>m-1$, and the given function $f$ of (2) is of class $C^{2}(x)$ for all $\left(x_{1}, \cdots, x_{m}\right)$ then the function $u(x, t)$, as defined by $(6)$, is of class $C^{2}(x, t)$, satisfies the initial conditions (2), and for $t \neq 0$ satisfies (1), while for $t=0$ it satisfies the limiting form (5).

\section{BibLIOGRAPHY}

[1] A. Weinstern, Sur le problème de Cauchy pour l'equation de Poisson et l'equation des ondes, Comptes Rendus Acad. Sci. Paris, 234 (1952) 2584-2585.

[2] A. Wernstern, On the Cauchy problem for the Euler-Poisson-Darboux equation, Bull. Amer. Math. Soc., 59 (1953) 454. On the wave equation of Euler-Poisson, Proc. 5th Symposium in Applied Mathematics, 1952 (McGraw-Hill, 1954), pp. 137-147.

[3] J. B. Diaz \& H. F. Weinberger, A solution of the singular initial value problem for the Euler-Poisson-Darboux equation, Proc. Amer. Math. Soc., 4 (1953) 703-718.

[4] J. B. Diaz \& G. S. S. LUdFord, On the Euler-Poisson-Darboux equation, integral operators, and the method of descent, Proceedings of the Conference on Differential Equations (dedicated to A. Weinstein on the occasion of his 60th birthday), University of Maryland (1956), pp. 73-89.

[5] O. BouzA, Lectures on the calculus of variations, (Reprint of edition 1904), G. E. Stechert Co., New York, 1931.

Institute for Fluid Dynamics and Applied Mathematics, University of Maryland

$$
\text { and }
$$

U. S. Army Ballistic Research Laboratories

Aberdeen Proving Ground, Maryland

Date Communicated: February 7, 1966 